Bangladesh Journal of Neuroscience 2016; Vol. 32 (1): 43-46

\title{
Cerebellar Ataxia, An Unusual Neurological Manifestation of Coeliac Disease- A Case Study
}

\author{
MD RAKNUZZAMAN ${ }^{1}$, ABDUL KADER SHEIKH ${ }^{3}$, HASAN ZHAHIDUR RAHMAN ${ }^{2}$, SK MAHBUB ALAM ${ }^{3}$, \\ SAIFULLAH AHTHESAM ${ }^{1}$, KAZI JANNAT ARA ${ }^{4}$, MD. RAFIQUL ISLAM ${ }^{2}$
}

\begin{abstract}
:
Coeliac disease was considered as a gluten sensitive enteropathy but now due to its wide clinical presentation is considered as multisystem autoimmune disorder. Ataxia with peripheral neuropathy is a rare manifestation of gluten sensitivity. The presence of glutenrelated immune markers in normal population however complicates the reliable diagnosis of gluten related neurological disorders and clinical improvement on gluten free diet can serve as a diagnostic tool for this disease. We report a case of sporadic progressive cerebellar ataxia with peripheral neuropathy with positive anti tissue transglutaminase (antitTG) antibodies and subtotal villous atrophy in duodenal biopsy. This case highlights an important diagnostic and therapeutic principle in management of late onset idiopathic ataxia.
\end{abstract}

Keywords: Coeliac disease, Ataxia, Anti TTG ab

\section{Case Presentation}

A 65-year-old diabetic, normotensive female housewife (fig-1) presented to us with five years history of gradually progressive unsteadiness of gait. He denied any motor weakness but complaints of tingling and numbness sensation in gloves and stocking pattern and complained of tremulousness of the hands with clumsiness in doing fine work and a tendency to sway to either side while walking, with occasional falls. Within 3 years of the onset of the illness, she was unable to walk without support but she had no speech difficulty and sphincter disturbance. She also complained of forgetfulness and difficulty in calculating and handling money but there was no history of fits, headache, hallucinations, delusions or any change in behavior or personality. She also complained of chronic diarrhea with 6-7 semisolid, non-foul smelling loose stools without blood or mucus per day for last 15 years. Diarrhea was not associated with vomiting, abdominal pain, loss of appetite, weight loss or fever. Patient also diagnosed as DM for last 5 years. She was on oral hypoglycemic agents with good compliance and well controlled. No history of consanguinity among her parents and there was no family history of any similar illness in the parents or siblings. Besides the habit of betel nut chewing she had no other addiction.

On examination, patient was fully conscious and alert. She was moderately anaemic, nonicteric with vitiligo over extremities and around lips. There was diffuse non tender thyromegaly. Her vitals and other systemic examination were normal. Higher mental functions were essentially normal (MMSE-27/30). All the cranial nerves were intact with normal fundus examination and full ocular movements without nystagmus. There was no motor weakness but all the deep tendon reflexes were diminished and planters were bilaterally flexor. Pain \& Temperature is lost in gloves and stocking pattern. Vibration is lost distally in both upper and lower limbs with positive Rhomberg's sign. She had bilateral cerebellar signs with in-coordination of finger nose test, impairment of heel shin test, and gait was broad based ataxic type.

On laboratory examination, complete blood count revealed $\mathrm{Hb}-8 \mathrm{gm} / \mathrm{dl}$, ESR-70 $\mathrm{mm} / 1^{\text {st }}$ hour with normal differentials, blood film shows microcytic hypochromic anaemia with elliptical, pencil and few

1. Resident Phase B Neurology, BSMMU

2. Professor of Neurology, BSMMU

3. Associate Professor of Neurology, BSMMU

4. Medical officer, BSMMU 
target cells, Serum ferritin $-8.88 \mu \mathrm{gm} / \mathrm{l}(\downarrow)$, Iron$35 \mu \mathrm{gm} / \mathrm{dl}(\downarrow)$, TIBC-411 $\mu \mathrm{gm} / \mathrm{dl}(\uparrow)$ Serum Vitamin B12- $326 \mathrm{pgm} / \mathrm{ml}$ ( Normal ), HbA1c- $6.8 \%$, Routine blood chemistry including blood sugar, LFT, RFT, Thyroid function test, and Serum electrolytes levels were within normal range. For evaluation of diarrhea stool r/e was done which was normal, anti TTG (tissue trans-glutaminase) antibody was positive $(68.5 \mathrm{U} / \mathrm{ml})$. Patient then undergone endoscopy of upper GIT and duodenal biopsy. It reveals chronic duodenitis with partial villous atrophy. MRI of brain showed cerebellar atrophy more marked over vermis (fig 2).
In view of a history of progressive cerebellar ataxia and peripheral neuropathy being accompanied by chronic diarrhea and a positive blood test for anti TTG (tissue trans-glutaminase) antibody and positive histopathology report of partial villous atrophy, a diagnosis of gluten sensitive disorder was confirmed. The patient was advised to follow a strict gluten free diet, following which her loose motions abated within a few days and was given some supportive management like iron and vitamin supplementation including vitamin $\mathrm{E}$ and she was discharged home with the advice to remain compliant with her diet plan and regular follow up.

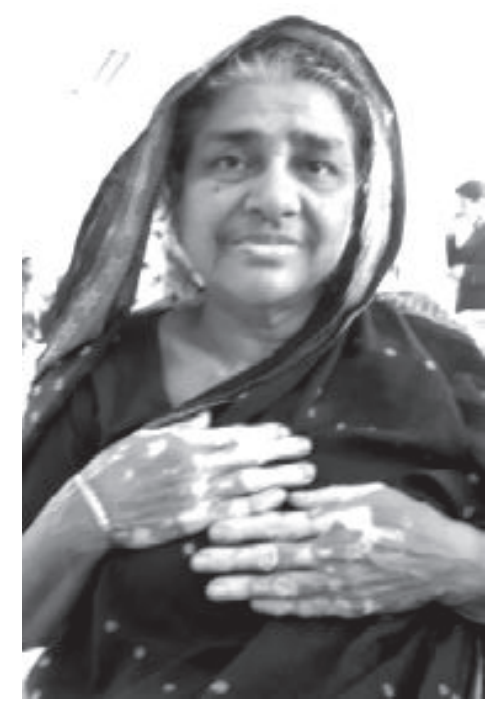

Fig-1: A 65 year female diabetic and normotensive patient
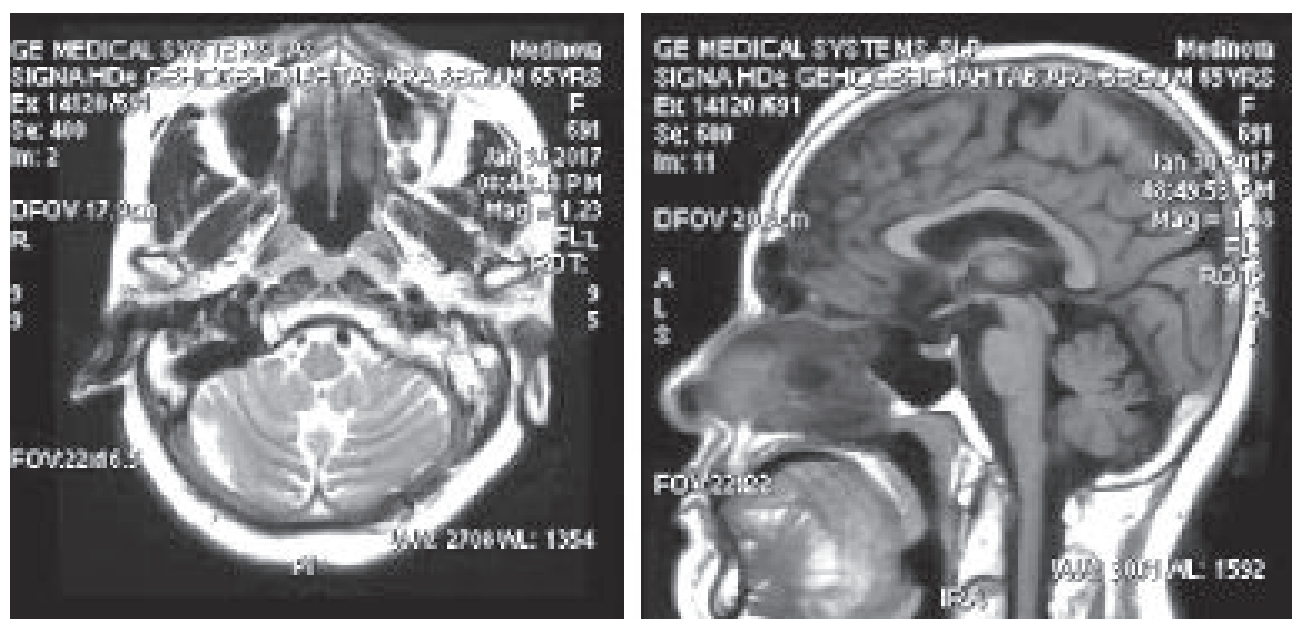

Fig-2: MRI of Brain axial \& saggital view showing bilateral cerebellar atrophy including vermis. 


\section{Discussion:}

Coeliac disease (CD) is a life-long gluten-sensitive autoimmune disease of the small intestine affecting genetically susceptible individuals worldwide and is seen more commonly in association with HLA DQ2 and DQ8 positivity ${ }^{1}$. CD individuals may present gastrointestinal symptoms, extraintestinal symptoms or no signs or symptoms. The classical symptoms include gastrointestinal-related symptoms such as diarrhea, steatorrhea and weight loss due to malabsorption. About $50 \%$ of CD patients present with extraintestinal or atypical symptoms, such as anemia, osteoporosis, dermatitis herpetiformis, neurological problems and dental enamel hypoplasia ${ }^{2,3}$. The prevalence of CD has been estimated to approximate $0.5 \%-1 \%$ in different parts of the world 4 . A study from north India reported a prevalence of 1 in 310 individuals ${ }^{5}$. Overall prevalence of gluten sensitivity is probably much higher, since Coeliac disease is frequently referred to as 'iceberg disease.

Neurological complications occur in about $8 \%-10 \%$ of patients with the disease ${ }^{6}$ including peripheral neuropathy 7,8 , progressive multifocal leucoencephalopathy ${ }^{9}$, cerebellar ataxia $6,10,11$, progressive myoclonic ataxia ${ }^{12,13}$, dementia ${ }^{14}$ and myopathy ${ }^{15}$.

Coeliac disease occurs in approximately $9 \%$ of patients with idiopathic cerebellar ataxia. Patients can present with ataxia of limb and/or dysarthria, oculomotor, sensory, or bladder dysfunction ${ }^{16}$. The ataxia occasionally improves after a prolonged gluten-free diet ${ }^{17}$. Ataxic patients also have a higher incidence of gluten sensitivity, defined as the presence of antibodies to gliadin or transglutaminase in the absence of histological evidence of coeliac disease ${ }^{16}$.

In patients with neurologic manifestations, gastrointestinal symptoms are detectable in 10\% of the cases only, but biopsy evidence of coeliac disease can be found in upto one-third of the patients. In our case, the patient had a history of chronic diarrhea but the intestinal biopsy also shows partial villous atrophy.

Serological tests used to confirm the diagnosis of gluten sensitivity include: IgA anti gliadin antibody,
IgA anti tissue trans-glutaminase (Anti-TTG) and anti endomysial antibodies. Anti TTG and endomysial antibodies are specific for enteropathy but these are often undetectable in patients with neurological manifestations but in our case this test also reveals positive at low titre. MR imaging shows cerebellar atrophy in around $60 \%$ of the cases, as similar lesion was found in the present case also (fig-2).

IVIg has had a beneficial effect in patients with sporadic cerebellar ataxia, in the context of GAD antibodies $^{18}$ and gluten sensitivity ${ }^{19}$ but has not yet been shown to work for the combination of ataxia and coeliac disease. It would be interesting to test plasmapheresis or corticosteroids, as an alternative to costly IVIg, and to ascertain whether all ataxic patients with coeliac disease eventually respond to therapeutic long-term immunosuppression.

The mechanisms for neurological manifestations of coeliac disease remain uncertain. The first proposed mechanism is malabsorption of nutrients exerting neurotrophic and neuroprotective effects ${ }^{20}$ but dietary gluten restriction and vitamin supplementation rarely improve the neurological deficits $^{8,12}$. Now an immunological mechanism is given priority in the literature: Anti gliadin Abs have been suggested to be directly or indirectly neurotoxic ${ }^{15}$.

\section{Conclusions:}

Neurologic manifestations can also be encountered in cases with gluten sensitivity and which may occur with or without gastrointestinal symptoms and can often be missed if it occurs in isolation. As gluten ataxia is a potentially treatable and reversible disorder, all patients presenting with sporadic, unexplained chronic cerebellar ataxia should be tested for serological evidence of gluten sensitivity. Patients testing positive for antigliadin or anti TG antibodies, should be put on a strict gluten free diet to reduce disability and arrest further disease progression. To the best of our knowledge, we report the first case of elderly lady with cerebellar atxia due to coeliac disease.

\section{References:}

1. Grossman G. Neurological complications of coeliac disease. What is the evidence? Pract Neurol. 2008;8:77-89. 
2. Rampertab SD, Pooran N, Brar P, Singh P, Green $\mathrm{PH}$. Trends in the presentation of celiac disease. Am J Med 2006; 119: 355.e9-355.14

3. Mäki M, Kallonen K, Lähdeaho ML, Visakorpi JK. Changing pattern of childhood coeliac disease in Finland. Acta Paediatr Scand 1988; 77: 408-412

4. Gujral N, Freeman HJ, Thomson A. Celiac disease: prevalence, diagnosis, pathogenesis and treatment. World J Gastroenterol. 2012;18(42):6036-59.

5. Sood A, Midha V, Sood N, Avasthi G, Sehgal A. Prevalence of celiac disease among school children in Punjab, north India. J Gastroenterol Hepatol. 2006;21:1622-25.

6. Finelli PF, McEntee WJ, Ambler M, et al. Adult celiac disease presenting as cerebellar syndrome. Neurology1980; 30:245-9.

7. Kaplan JG, Pack D, Horoupian D, et al. Distal axonopathy associated with chronic gluten enteropathy: a treatable disorder. Neurology 1988;38:642-5.

8. Kelkar P, Ross MA, Murray J. Mononeuropathy multiplex associated with celiac sprue. Muscle Nerve 1996;19:234-6.

9. Kepes JJ, Chou SM, Price LW. Progressive multifocal leucoencephalopathy with 10-year survival in a patient with nontropical sprue. Neurology 1975;25:1006-12.

10. Ward ME, Murphy JT, Greenberg GR. Celiac disease presenting with cerebellar degeneration with normal vitamin E status. Neurology 1985;35:1199-201.

11. Mauro A, Orsi L, Mortara P, et al. Cerebellar syndrome in adult celiac disease with vitamin E deficiency. Acta Neurol Scand 1991; 84:167-70.
12. Lu CS, Thompson PD, Quinn NP, et al. Ramsay Hunt syndrome and coeliac disease: a new association? Mov Disord 1986;1:209-19.

13. Bathia KP, Brown P, Gregory R, et al. Progressive myoclonic ataxia associated with coeliac disease.

The myoclonus is of cortical origin, but the pathology is in the cerebellum. Brain 1995;118:1087-93.

14. Collin P, Pirttila T, Nurmikko $T$, et al. Celiac disease, brain atrophy, and dementia. Neurology 1991;41:372-5.

15. Hall WH. Proximal muscle atrophy in adult celiac disease. Am J Dig Dis 1968;13: 697-704.

16. Hadjivassiliou M, Grunewald R, Sharrack B, et al. Gluten ataxia in perspective: epidemiology, genetic susceptibility and clinical characteristics. Brain 2003; 126: 685-91.

17. Pellecchia MT, Scala R, Perretti A, et al. Cerebellar ataxia associated with subclinical celiac disease responding to gluten-free diet. Neurology 1999; 53: 1606-08.

18. Vianello M, Tavolato B, Armani M, Giometto B. Cerebellar ataxia associated with antiglutamic acid

decarboxylase autoantibodies. Cerebellum 2003; 2: 77-79.

19. Burk K, Melms A, Schulz JB, Dichgans J. Effectiveness of intravenous immunoglobin therapy in

cerebellar ataxia associated with gluten sensitivity. Ann Neurol 2001; 50: 827-28.

20. Cooke WT, Smith WT. Neurological disorders associated with adult coeliac disease. Brain 1966;89:683-722. 